\title{
Urgences
}

\section{Obscures constructions : tentatives et expérimentations timides}

\section{Marie Bélisle}

Numéro 22, janvier 1989

Octet

URI : https://id.erudit.org/iderudit/025502ar

DOI : https://doi.org/10.7202/025502ar

Aller au sommaire du numéro

Éditeur(s)

Urgences

ISSN

0226-9554 (imprimé)

1927-3924 (numérique)

Découvrir la revue

Citer ce document

Bélisle, M. (1989). Obscures constructions : tentatives et expérimentations timides. Urgences, (22), 15-22. https://doi.org/10.7202/025502ar d'utilisation que vous pouvez consulter en ligne.

https://apropos.erudit.org/fr/usagers/politique-dutilisation/ 


\section{OBSCURES CONSTRUCTIONS:}

\section{TENTATIVES ET EXPÉRIMENTATIONS TIMIDES Marie Bélisle}

Marie Bélisle n'est pas (ne sera sans doute jamais) informaticienne. Cependant ce bon vieux désir de posséder, comprendre, contrôler mes «outils de production» et le plaisir constant de manipuler du langage (naturel ou non) m'ont poussée vers l'informatique, m'ont amenée à expérimenter cette autre forme de création. Car c'est bien de création qu'il s'agit: du projet à l'objet, de semblables erreurs, hésitations, corrections, une semblable recherche de l'efficace.

D'une part, depuis 20 ans, une fascination pour les mathématiques: la certitude d'une parenté entre l'abstrait du poème et l'abstrait du théorème, entre le système du texte et le système des nombres: "ll y a l'écriture dans l'équation.»"

D'autre part, il y a 5 ans, l'achat d'un ordinateur personnel et la découverte progressive de l'apport qu'il fournit au travail d'écriture: traitement de texte et bases de données d'abord (ce qui n'est pas rien), programme de «lecture», de combinatoire, de génération aléatoire de texte, etc. ensuite.

Bref, depuis quelques temps, l'irruption dans mon écriture d'éléments mathématiques (au niveau de la structure et/ou à celui du lexique $)^{2}$, et le recours à l'ordinateur comme «assistant à la réalisation» des textes ${ }^{3}$. Et, depuis peu, l'amorce d'un travail spécifiquement informatique: la conception de programmes.

Résultats: l'exploration, par l'intermédiaire de langages de programmation «accessibles» (Logo, Pascal), de manipulations textuelles informatisées élémentaires et l'utilisation des produits de ces manipulations comme matière première (seconde?) ou comme matrice d'écriture.

D'évidence, il n'y a là, pour l'instant, rien de très raffiné (informatiquement parlant). II n'y a qu'une timide tentative de démonstration de la possibilité, pour une novice, d'utiliser un ordinateur autrement que comme une machine à écrire perfectionnée...

À titre d'exemples du type de travail que l'ordinateur me permet, malgré mon manque flagrant de connaissances et d'expérience: 
des textes produits avec l'«assistance» d'un petit générateur d'acrostiches (que j'ai réalisé en LOGO (1987)) et du programme Quenine de Bernard Magné.

\section{LES OUTILS}

Le programme Acrostiche choisit au hasard des mots ou syntagmes dans une banque alphabétique intégrée; il existe en deux versions: la première moins élaborée, ne fournit que des mots (ou syntagmes courts). Dans cette version, l'écriture est à faire, à partir de la matière lexicale fournie. L'intérêt vient alors du traitement ultérieur (informatisé ou non - que je ferai subir à cette matière, du travail textuel qui s'amorcera/se poursuivra. C'est cette version qui m'a donné (à partir des lettres $\mathrm{O}, \mathrm{C}, \mathrm{T}, \mathrm{E}, \mathrm{T}$ ) les deux séries traitées ensuite par le programme Quenine. La deuxième version fournit du texte plus "fini», directement utilisable. Dans cette version, l'écriture a donc été faite, à la ponctuation près, au moment même de la programmation: c'est à ce moment que le travail textuel a été le plus complexe.

Le programme Quenine permet de faire permuter un nombre donné d'éléments de façon à ce que chacun de ceux-ci occupe au moins une fois et une seule fois toutes les positions prévues par leur nombre même. Ainsi, dans une quenine d'ordre 5, chacun des éléments de la série occupera successivement cinq positions; il en résultera cinq «arrangements" différents de la série. La forme de la quenine, élaborée par Raymond Queneau, découle de celle de la sextine du troubadour Arnaud Daniel ${ }^{4}$. 


\section{LE TRAITEMENT}

Avec la première version d'Acrostiche, j'ai obtenu:

$1^{\circ}$ série: Organique, C'est-à-dire, Tacitement, Écarlate, Toutefois;

$2^{e}$ série: Obscurément, Calme, Tragique, Étrange, Texte.

Traitées par Quenine, et permutant l'une à l'inverse de l'autre, les deux séries ont constitué la structure de strophes suivante:

C'est-à-dire Obscurément

Écarlate Calme

Toutefois Tragique

Tacitement Étrange

Organique Texte

Écarlate Texte

Tacitement

Organique

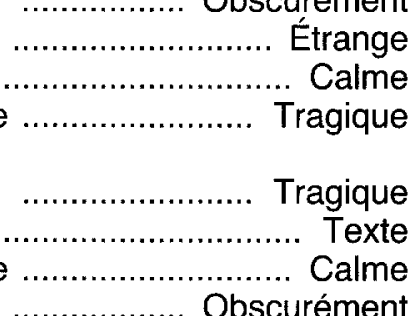

Toutefois

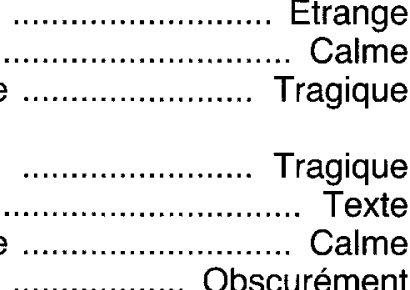
C'est-à-dire

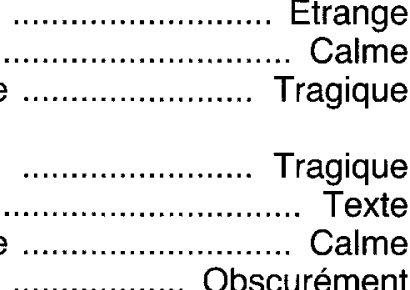

Tacitement

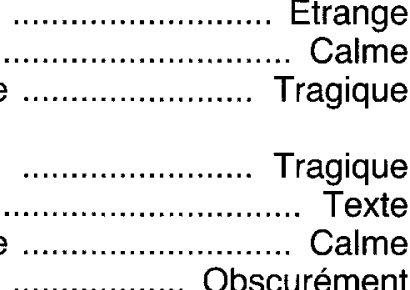

Toutefois

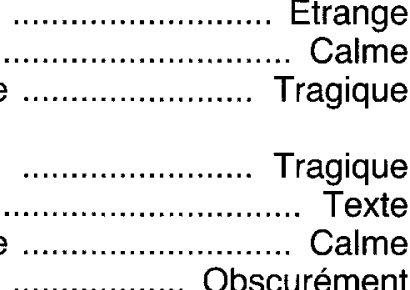

C'est-à-dire

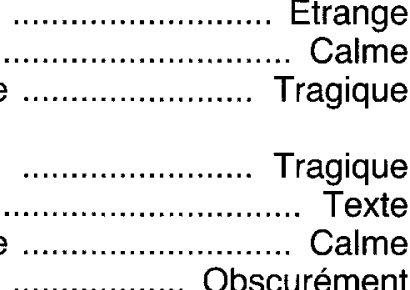
Organique Écarlate Obscurément Étrange

Toutefois

Organique Étrange

Écarlate Tragique C'est-à-dire Obscurément

Tacitement Texte Calme

Organique

C'est-à-dire Calme

Tacitement

Écarlate Étrange Toutefois Texte Tragique Obscurément

Les mots des séries ont alors été utilisés respectivement comme début et fin de ligne; entre eux ont été glissés (à la main ${ }^{5} .$. ) d'autres éléments de texte et de ponctuation. Résultat: le texte 1. 


\section{LES PRODUITS}

1.

C'est-à-dire que la mémoire obscurément écarlate et vive se constitue, dans le calme; toutefois, parallèlement, subsiste le tragique.

tacitement périphérique: c'est l'interface étrange, organique, entre l'être et le texte.

Écarlate donc, la mémoire joue l'écart: le texte, tacitement, transmissible, obscurément organique, s'écrit comme une lettre étrange; toutefois, le geste se développe, calme, c'est-à-dire tragique.

Tacitement la conscience du tragique, toutefois subreptice, travestit le texte c'est-à-dire le subvertit malgré le calme organique apparent; dans la mémoire obscurément écarlate s'imprime cette figure étrange.

Toutefois cette figure, même étrange, organique, n'atteint pas l'écran tragique, écarlate par l'absurde, où obscurément c'est-à-dire obstinément le texte tacitement s'écrase, saisi, calme.

Organique comme vivante végétale et calme c'est-à-dire verte, la lumière étrange tacitement se substitue au texte écarlate: ainsi s'opère la perte du tragique; toutefois, l'être s'attache à la lettre, obscurément. 
Avec la deuxième version du programme Acrostiche, le texte s'est directement constitué, la banque alphabétique intégrée contenant des syntagmes complexes et relativement autonomes. Résultat: le texte 2 .

2.

Obscurément comme un glissement, car le casse-tête se construit, tout s'éloigne;

et l'écart s'ébauche, très très pâle pourtant. 


\section{OBSCURES NARCISSIQUES}

À titre de bonus, deux textes construits avec les mêmes programmes, selon les mêmes modèles que les précédents, mais transp(r)osés.

Devinette: quel mot a servi de générateur aux acrostiches traités?

1.

Aventure du mot à mot: in abstracto s'inscrit, alors étrange comme un nombre rare, réellement, l'ombre identique, mimétique de l'algorithme; exactement.

In abstracto apparaît, exactement réellement narcissique, le jeu du mot à mot mimétique, comme une recherche de l'identique étrange(r), mais réfléchi jusqu'alors: aventure en quelque sorte de l'être rare.

Réellement se nomme la lettre rare: étrange occurrence saisie exactement: aventure de la mémoire comme matière alors mimétique, représentée par le mot à mot, in abstracto par effort d'identique.

Étrange inquiétude du dédoublement: identique, mimétique, le signe étouffé s'est fait rare, in abstracto il s'est dissous dans le mot à mot; aventure des spéculations exactement réellement fractionnées alors.

Mimétique, la fiction devient alors aventure du sens propre, identique réellement, autobiographique exactement, in abstracto multipliée par le chiffre même rare: étrange dénominateur commun du mot à mot.

2.

Même le texte bascule. À quelques lettres près, réellement cela s'inscrit: ici l'ordre s'estompe. Ensuite l'extrême errance s'esquisse.

1. Michel Cassé «Épreuves d'écriture», Les immatériaux, Paris, Éditions du Centre Georges Pompidou, 1985, p. 55.

2. Voir "Les trahisons de l'hyperbole", paru dans Urgences no 21.

3. Voir "Chronique acronymique", à paraître dans la NBJ.

4. Pour plus de détails sur la quenine, voir: Oulipo, Atlas de littérature potentielle, Paris, Gallimard, coll. «Idées», 1981, pp. 243-248.

5. La main sur le clavier, bien sûr! 


\section{MÉMOIRE VIVE}

[L'informatique) ouvre un champ de renouvellement illimité à la création du texte, sans jamais entraver les pulsions du désir. Le traitement de texte est lui-même source de désir, puisqu'il est une invitation ludique à l'écriture, source de paresse amoureuse. II lui confère un pouvoir érotique.

Enfin, j'aime à penser que le traitement de texte est aussi gardien de l'écriture. À cause de son infinie malléabilité, son abord est difficile à ceux qui ignorent les passions qu'elle suscite. Devant les phrases qui se construisent ou s'effacent au moindre souffle électronique, l'écriture devient leurre, vertige. Elle se heurte à la phrase insaisissable, au substantif pervers, au verbe irréductible, sans possibilité de les maîtriser. Elle devient course aux chevaux sauvages dans une Sardaigne de songe, au sommet du relief tabulaire (tabulateur) où le nuraghe rejoint le nuage.

II peut aussi malheureusement permettre à ceux qui savent écrire et qui n'ont strictement rien à dire d'accumuler des milliers de pages sans problème, mais cela, n'importe quelle autre méthode d'écriture l'autorise aussi.

Philippe Curval, 6, p. 63 


\section{MÉMOIRE VIVE}

Ce qui est amusant dans l'informatique, c'est le mal que se donnent les génies de Silicon Valley pour reconstituer les formes les plus anciennes de l'écriture: la note de bas de page, le titre courant, la glose, l'équation, la figure dans le texte, la page à l'écran. Toute une technique se trouve ainsi mise au service des vieux métiers de l'archivage, de l'indexation, de la copie, de l'écriture. Tout le monde devient scribe. Triomphe et généralisation de Gutenberg. L'audiovisuel disparaît, absorbé par cet hybride: la page à l'écran. Même les analphabètes sont au clavier.

Bruno Latour, 6, p. 55 